\section{Check for updates}

Cite this: J. Mater. Chem. A, 2019, 7, 11814

\title{
Unravelling mass transport in hierarchically porous catalysts $\uparrow$
}

\author{
Mark A. Isaacs, (DD a Neil Robinson, (iD ${ }^{b}$ Brunella Barbero, ${ }^{c}$ Lee J. Durndell, ${ }^{d}$ \\ Jinesh C. Manayil, ${ }^{C}$ Christopher M. A. Parlett, (D) ${ }^{\text {ef }}$ Carmine D'Agostino, (D) *e \\ Karen Wilson (D) ${ }^{g}$ and Adam F. Lee (D) *g
}

\begin{abstract}
Bio-derived platform chemicals and fuels are important for the development of sustainable manufacturing. However, their efficient production from biomass necessitates new catalysts and processes optimised for the selective transformation of large molecules. Mesoporous and hierarchically porous functional materials are promising catalyst candidates for biomass valorisation, but quantitative relationships between pore dimensions/connectivity, mass transport, and corresponding catalytic performance are poorly defined. A family of hierarchical macroporous-mesoporous SBA-15 sulfonic acids were prepared with tunable macropore diameters for carboxylic acid esterification. Turnover frequencies for long-chain (palmitic and erucic) acids were proportional to macropore diameter ( $\leq 370 \mathrm{~nm}$ ), whereas propanoic acid esterification was independent of macropore size. Pulsed field gradient NMR diffusion experiments reveal that larger macropores enhance esterification of bulky carboxylic acids by conferring superior pore interconnectivity and associated mass transport.
\end{abstract}

Received 19th February 2019

Accepted 8th April 2019

DOI: $10.1039 / c 9 t a 01867 \mathrm{k}$

rsc.li/materials-a promote esterification $^{9}$ and selective alcohol oxidation ${ }^{\mathbf{1 0}}$ over sulfonic acid and Pd mesoporous SBA-15 silicas respectively, $\mathrm{CO}_{2}$ hydrogenation over $\mathrm{Cu}$ alumina, ${ }^{11}$ and transesterification over $\mathrm{Mg}-\mathrm{Al}$ hydrotalcites. ${ }^{12}$ This is conceptually akin to fractal networks in Nature, ${ }^{13}$ wherein the transport of matter is optimised by rapid convective flow through large channels, that in turn supply a network of smaller channels through which diffusion is constrained. Although chemical transport through hierarchical pore networks is extensively modelled, natureinspired design principles ${ }^{\mathbf{1 4}}$ have yet to be implemented to synthesise materials with architectures 'tailored for purpose'.

Considering heterogeneous catalysis, simulations suggest that in the Knudsen diffusion regime, where reactants/products can enter/exit mesopores but experience attendant diffusion limitation, hierarchical bimodal pore networks can significantly improve active site accessibility, with larger pores acting as 'molecular superhighways'15 to accelerate transport from the bulk media to mesopore domains. ${ }^{16-19}$ In practice, such enhanced accessibility is also facilitated by truncation of mesopore channels in ordered hierarchical porous solids (commonly prepared by dual-templating routes) compared with their mesoporous counterparts; ${ }^{20,21}$ shorter mesopores may mitigate any attendant diffusional resistance. The interdependence of pore hierarchy and individual pore dimensions in experimental studies hampers validation of transport models and necessitates direct measurement of diffusion and reaction in systematically related families of hierarchical porous solids.

Routes to interpenetrating bimodal macro-mesopore networks using microemulsion colloidal polystyrene microspheres or co- 
surfactant templating routes are particularly attractive for liquid phase heterogeneous catalysis using bulky substrates. Hierarchical macroporous-mesoporous silicas offer great flexibility in pore size and connectivity through the choice of soft/hard surfactant template, use of porogens, and hydrothermal processing. ${ }^{20,22-25}$ However, in the context of catalysis, systematic studies of porosity are largely confined to mesoporous silicas, wherein e.g. pore-expansion increased the turnover frequency (TOF) of sulfonic acid functionalised SBA-15 and KIT-6 for carboxylic acid esterification, ${ }^{26}$ or hierarchically porous silicas in which only the mesopore diameter was varied for Fischer-Tropsch synthesis. ${ }^{27}$ In all cases, improved mass transport is inferred from superior catalytic performance. Nevertheless, very few experimental studies have correlated molecular diffusion through mesopores and corresponding reactivity, ${ }^{28,29}$ and to our knowledge diffusion has never been experimentally measured for an ordered hierarchical porous catalyst. The mechanism by which macropores improve reactivity in hierarchically porous catalysts remains unclear, but is amenable to interrogation by pulsed field gradient (PFG) NMR, widely applied to elucidate transport phenomena within liquidsaturated porous media. ${ }^{30-32}$

Here we assess the impact of macropore size on molecular diffusion and carboxylic acid esterification for propylsulfonic acid functionalised, hierarchical macroporous-mesoporous SBA-15 $\left(\mathrm{PrSO}_{3} \mathrm{H} / \mathrm{MM}-\mathrm{SBA}-15\right)$ catalysts. PFG NMR is used to probe diffusive restriction and pore connectivity within these catalyst frameworks. Larger macropores reduce pore network tortuosity and increase diffusion through mesopore channels, accounting for the $>4$-fold increase in TOF observed for palmitic and erucic acid esterification with methanol.

\section{Experimental}

\section{Polystyrene nanosphere synthesis}

A family of monodispersed polystyrene nanospheres (200, 300, 400,500 , and $600 \mathrm{~nm}$ diameter) were prepared using a modified literature protocol. ${ }^{33}$ Styrene $\left(50 \mathrm{~cm}^{3}\right.$ for $200 \mathrm{~nm}$ beads increasing linearly to $150 \mathrm{~cm}^{3}$ for $600 \mathrm{~nm}$, Aldrich 99\%) was washed five times with aqueous $\mathrm{NaOH}(0.1 \mathrm{M}, 1: 1$ by volume) and five times with deionized water $(1: 1$ by volume) to remove the 4-tert butylcatechol polymerisation inhibitor; larger styrene volumes yielded larger beads (Fig. S1 $\dagger$ ). Washed styrene was then added to $\mathrm{N}_{2}$ degassed, deionised water $\left(1000 \mathrm{~cm}^{3}\right)$ at $80^{\circ} \mathrm{C}$, to which aqueous $\mathrm{K}_{2} \mathrm{~S}_{2} \mathrm{O}_{8}\left(0.24 \mathrm{M}, 50 \mathrm{~cm}^{3}\right.$, Aldrich $\left.\geq 99 \%\right)$ was added dropwise with stirring (300 rpm). Reactions were stopped after $22 \mathrm{~h}$, the resulting solid products recovered by filtration, and colloidal crystal assembly induced by centrifugation (Heraeus Multifuge X1 with Thermo Fiberlite F15-8x50cy FixedAngle Rotor, operated at $8000 \mathrm{rpm} / 7441 \mathrm{~g}$ for $1 \mathrm{~h}$ ). The resulting highly ordered, polystyrene nanosphere crystalline matrices were ground to fine powders.

\section{Hierarchically porous SBA-15}

Hierarchically porous silicas were prepared by a modified true liquid crystal templating technique incorporating a range of polystyrene nanospheres as macropore-directing hard templates. ${ }^{34}$ Pluronic P123 (2 g, Aldrich average $M_{\mathrm{n}} \sim 5800$ ) was sonicated with $\mathrm{HCl}$-acidified deionised water $(2 \mathrm{~g}, \mathrm{pH} 2)$ at $40^{\circ} \mathrm{C}$ to form a homogeneous gel. Tetramethoxysilane $\left(4.08 \mathrm{~cm}^{3}\right.$, Acros 99\%) was added to the gel and rapidly stirred for $5 \mathrm{~min}$ (800 rpm) until a homogeneous liquid formed. Immediately following this phase change, polystyrene nanospheres of a particular size ( $6 \mathrm{~g}$, independent of bead diameter, as a fine powder) were added with agitation (100 rpm) for $1 \mathrm{~min}$. The resulting viscous, homogeneous mixtures were heated in vacuo (100 mbar) at $40{ }^{\circ} \mathrm{C}$ for $2 \mathrm{~h}$ to remove reactively-formed methanol. The solids thus obtained were exposed to air at room temperature for $24 \mathrm{~h}$ to complete condensation of the silicate precursor, and then calcined at $550{ }^{\circ} \mathrm{C}$ for $6 \mathrm{~h}$ (ramp rate $5{ }^{\circ} \mathrm{C} \min ^{-1}$ ) to remove organic templates.

\section{Acid functionalisation}

The preceding hierarchically porous silicas were functionalised with propylsulfonic acid by an alkoxide grafting method. In each case, silica $(1 \mathrm{~g})$ was added to a $100 \mathrm{~mL}$ round-bottomed flask with anhydrous toluene $(30 \mathrm{~mL})$ under $\mathrm{N}_{2}$ at $90{ }^{\circ} \mathrm{C}$ with stirring (700 rpm). A (3-mercaptopropyl)trimethoxysilane thiol precursor $(1 \mathrm{~mL})$ was subsequently added and the reaction mixture stirred for $24 \mathrm{~h}$. The resulting solid was filtered, washed with ethanol, and dried at $80{ }^{\circ} \mathrm{C}$ overnight. Surface thiols were then oxidised to sulfonic acids $(1 \mathrm{~g}$ solid in $20 \mathrm{~mL}$ of $30 \mathrm{vol} \%$ $\mathrm{H}_{2} \mathrm{O}_{2}$ ) with stirring $(400 \mathrm{rpm})$ overnight. Propylsulfonic acid functionalised materials were recovered, washed with deionised water and ethanol, and dried overnight at $80^{\circ} \mathrm{C}$.

\section{Material characterisation}

Powder XRD was performed on a Bruker D8 ADVANCE fitted with a $\mathrm{Cu} \mathrm{K} \mathrm{K}_{\alpha}$ X-ray source with a Ni filter and LynxEYE 192channel high speed strip detector. Wide angle scans were performed between $10-80^{\circ}$ with a step size of $0.02^{\circ}$ and dwell time of $0.6 \mathrm{~s}$, and low angle scans between $0.5-8^{\circ}$ with a step size of $0.01^{\circ}$ and a dwell time of $1 \mathrm{~s}$. Crystalline phases were determined by Rietveld refinement using Profex v3.11.1, and crystallite sizes determined using the Scherrer equation. $\mathrm{N}_{2}$ adsorption isotherms were measured using a Quantachrome 4000 surface area analyser. Surface areas and pore size distributions were determined through application of the BET and $\mathrm{BJH}$ equations respectively to the recorded isotherms. XPS analysis was performed using a Kratos Axis HSi spectrometer equipped with a monochromated $\mathrm{Al} \mathrm{K}_{\alpha}(1486.8 \mathrm{eV})$ X-ray source and charge neutraliser, operating $<10^{-9}$ Torr. Survey spectra were recorded with a pass energy of $160 \mathrm{eV}$, and high-resolution spectra with a pass energy of $40 \mathrm{eV}$. Spectra were Shirley background-subtracted, calibrated to adventitious carbon $(284.8 \mathrm{eV})$, and fitted using Casa v2.3.15. S 2p peaks were fitted using a Gaussian-Lorentzian $(70: 30)$ lineshape and a doublet separation of $1.16 \mathrm{eV}$. Sulfur loadings were determined by CHNS analysis using a Thermo Flash 2000 organic elemental analyser calibrated against a sulphanilamide standard. SEM was performed using a JEOL 7800F Prime FEG SEM fitted with a BSE and SE detector. Samples were affixed to carbon tape, and images recorded at a probe current of $3 \mathrm{~mA}$ and an accelerating 
voltage between 1-15 kV. STEM images were recorded using an aberration-corrected JEOL 2100F STEM with an accelerating voltage of $200 \mathrm{kV}$, a HAADF detector and Gatan bright field camera. Electron micrographs were processed with ImageJ 1.46r, and particle sizes determined from samples of $>200$ particles.

\section{Catalytic testing}

Esterification reactions were performed in a $50 \mathrm{~mL}$ roundbottomed flask at $90{ }^{\circ} \mathrm{C}$ with stirring (700 rpm). $25 \mathrm{mg}$ of catalyst was tested with $5 \mathrm{mmol}$ of propanoic acid, palmitic acid, or erucic acid, and $12.5 \mathrm{~mL}$ methanol with $0.1 \mathrm{mmol}$ dihexylether as an internal standard. $0.25 \mathrm{~mL}$ aliquots were periodically removed, diluted with methanol, and analysed using a Varian 450 GC with 8400 autosampler fitted with a ZB-50 column.

\section{PFG NMR}

Pulsed-field gradient (PFG) NMR diffusion measurements were performed to assess the diffusive behaviour of liquids in the unrestricted bulk and within $\mathrm{PrSO}_{3} \mathrm{H} / \mathrm{MM}-\mathrm{SBA}-15$ catalysts. Samples for NMR analysis were prepared according to the following procedure: $\mathrm{PrSO}_{3} \mathrm{H} / \mathrm{MM}-\mathrm{SBA}-15$ exhibiting $150 \mathrm{~nm}$ and $430 \mathrm{~nm}$ macropores were pressed into $1 \mathrm{~cm}$ diameter pellets. The pellets were broken apart into approximately $4 \mathrm{~mm}$ $\times 4 \mathrm{~mm} \times 2 \mathrm{~mm}$ pieces and dried at $105{ }^{\circ} \mathrm{C}$ for $12 \mathrm{~h}$. The catalysts were soaked in excess cyclohexane (Sigma Aldrich, $\geq 99.5 \%$ ) or dodecane (Fischer Scientific, $\geq 99 \%$ ) for at least $48 \mathrm{~h}$ under ambient conditions, with each liquid absorbed through capillary imbibition. Prior to NMR analysis the saturated materials were removed from the liquids and rolled over presoaked filter paper; this process removed any excess liquid on the outer surface of the pellets without drawing liquid from the pores. The saturated materials were then transferred to sealed $5 \mathrm{~mm}$ NMR tubes to a sample depth of $\sim 10 \mathrm{~mm}$.

${ }^{1} \mathrm{H}$ PFG NMR experiments were performed using a Bruker DMX 300 NMR spectrometer equipped with a $7.1 \mathrm{~T}$ magnet (300.13 $\mathrm{MHz}$ for ${ }^{1} \mathrm{H}$ ) and a Bruker Diff30 diffusion probe capable of producing pulsed magnetic field gradients up to 11.6 $\mathrm{T} \mathrm{m}^{-1}$. Samples were placed at the centre of the radiofrequency coil and left for at least 15 minutes to thermally equilibrate. Diffusion of unrestricted liquids was analysed using the pulsed gradient stimulated echo (PGSTE) sequence (Fig. 1a). ${ }^{35}$ To minimise the effects of background magnetic field gradients (so-called internal gradients ${ }^{36}$ ) the diffusion of liquids confined to the porous catalysts was analysed using the alternating pulsed gradient stimulated echo (APGSTE) pulse sequence (Fig. 1b). ${ }^{37}$ In each case, measurements were performed by holding the gradient pulse duration $\delta$ constant and linearly varying the magnetic field gradient strength $g$. Trapezoidal gradient pulses were used to ensure reproducible pulse shapes across all values of $g$. The recycle time was $2 T_{1}$ (as measured using the inversion recovery method) and 2 dummy scans were used to equilibrate the sample magnetisation before the acquisition of each data point. All measurements were performed at $20 \pm 0.1{ }^{\circ} \mathrm{C}$ and under ambient pressure. A summary a)

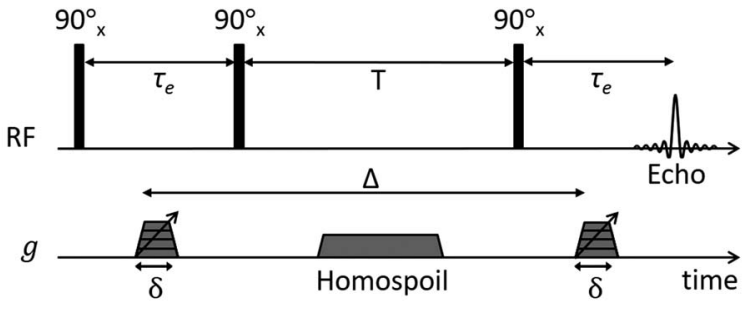

b)

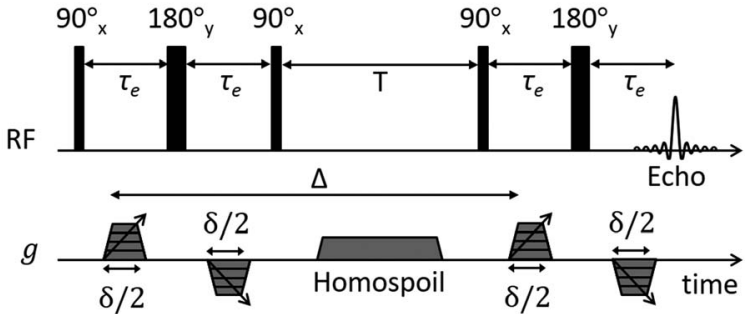

Fig. 1 NMR diffusion pulse sequence diagrams for (a) PGSTE and (b) APGSTE experiments. Radiofrequency (RF axis) pulses are indicated by vertical bars; thin and thick bars represent $90^{\circ}$ and $180^{\circ}$ RF pulses respectively. Gradient pulse timings ( $g$ axis) are specified according to the notation of Tanner. ${ }^{38}$ Trapezoidal gradient pulses of incremental magnitude $g$ are shown with effective pulse durations $\delta$ and $\delta / 2$ for the PGSTE and APGSTE sequences, respectively. Homospoil gradients are also shown and are applied during the longitudinal storage period $T$ to remove any residual transverse magnetisation. The observation time is $\Delta$.

Table 1 Summary of typical PFG NMR acquisition parameters

\begin{tabular}{lll}
\hline PFG NMR parameters & $\begin{array}{l}\text { Unrestricted } \\
\text { liquids }\end{array}$ & $\begin{array}{l}\text { Liquids in } \\
\text { hierarchical } \\
\text { catalysts }\end{array}$ \\
\hline Pulse sequence & PGSTE & APGSTE \\
Observation time, $\Delta / \mathrm{ms}$ & 50 & $25-200$ \\
Effective gradient pulse duration, $\delta / \mathrm{ms}$ & 1 & 1 \\
Maximum gradient strength/G cm ${ }^{-1}$ & 140 & 900 \\
Spin echo time, $\tau_{\mathrm{e}} / \mathrm{ms}$ & 3.2 & 2.7 \\
Homospoil gradient duration $/ \mathrm{ms}$ & 10 & 5 \\
No. of gradient steps & 16 & 32 \\
No. of scans & 16 & 64 \\
& &
\end{tabular}

of the typical PFG NMR acquisition parameters used is reported in Table 1.

\section{Results and discussion}

\section{Synthesis of macroporous SBA-15}

Macropore size control was achieved through the synthesis of monodispersed polystyrene nanospheres of tunable diameter (200 to $580 \mathrm{~nm}$ ) as sacrificial hard templates and their subsequent compaction into a colloidal crystal assembly. Ordered, hexagonal close-packed mesoporous SBA-15 silica networks were subsequently formed throughout the crystal assembly by a true liquid crystal templating method. ${ }^{34}$ Calcination resulted in hierarchically macroporous-mesoporous silicas (MM-SBA15) with uniform macropore diameters of 150 to $430 \mathrm{~nm}$ 
(proportional to template size) shown in Fig. 2; macropore shrinkage relative to the polystyrene template was consistent with previous reports for hierarchically porous alumina. ${ }^{39} \mathrm{~N}_{2}$ porosimetry of the as-prepared porous frameworks revealed a common type-IV isotherm with $\mathrm{H} 1$ hysteresis indicative of mesoporous SBA-15, with common BJH mesopore diameters of $4 \mathrm{~nm}$ (Fig. S2 $\dagger$ ). Low angle XRD confirmed the $\mathrm{d}_{10}$ reflection characteristic of $p 6 \mathrm{~mm}$ SBA-15 was present for all materials (Fig. S3†).

The preceding silica frameworks were functionalised with a common, and uniformly distributed, propylsulfonic acid $\left(\mathrm{PrSO}_{3} \mathrm{H}\right)$ loading $(\sim 1 \mathrm{wt} \% \mathrm{~S}$, Table $\mathrm{S} 1 \dagger)$ to yield a family of hierarchically porous solid acid catalysts $\left(\mathrm{PrSO}_{3} \mathrm{H} / \mathrm{MM}-\mathrm{SBA}-15\right)$. S 2p XP spectra (Fig. S4 $\dagger$ ) confirmed complete oxidation of the thiol precursor, and $\mathrm{N}_{2}$ porosimetry confirmed retention of the parent mesopore networks following $\mathrm{PrSO}_{3} \mathrm{H}$ functionalisation (Fig. S5 $\dagger$ ). The density of sulfonic acid groups was approximately independent of macropore diameter $\left(0.69 \mathrm{~nm}^{-2} \pm 0.05\right)$, and in a regime where the strongest acidity obtains due to lateral interactions between neighbouring sulfonic acid head groups. ${ }^{40}$ HAADF-STEM showed that mesopores were highly ordered in all cases, with a common mesopore diameter and macropore/mesopore interface (Fig. S6†).

\section{Catalytic enhancement through macropore tuning}

The impact of macropore diameter on the activity of $\mathrm{PrSO}_{3} \mathrm{H}$ / MM-SBA-15 catalysts was studied for free fatty acid (FFA) esterification with methanol as a function of chain length. In the case of propanoic acid, turnover frequencies (TOFs) per sulfonic acid site were independent of macropore size (and similar to that reported for mesoporous $\left.\mathrm{PrSO}_{3} \mathrm{H} / \mathrm{SBA}-15\right),{ }^{26}$ whereas those for bulky palmitic $\left(\mathrm{C}_{16: 0}\right)$ and erucic $\left(\mathrm{C}_{22: 1}\right)$ acids both significantly increased with macropore size (Fig. 3). For palmitic acid, per site activities increased four-fold as the macropores expanded from 150 to $430 \mathrm{~nm}$, while negligible activity was observed for the unsaturated, bent erucic acid for $150 \mathrm{~nm}$ macropores. Although erucic acid can readily access $150 \mathrm{~nm}$ macropores, only $2-8 \%$ of all active sites reside in the macropores (Table $\mathrm{S} 1 \dagger$ ) and hence negligible catalysis occurs within them. The question arises as to why increasing the macropore size promotes erucic acid esterification. A plausible explanation is that the increased pore connectivity for larger macropores (observed by PFG NMR below) is at least partially associated with break-up of the mesopore channel network, ${ }^{20,21}$ which in turn increases the number of mesopore openings and opportunities for the carboxylate group to react over active sites at mesopore entrances. This is akin to the mechanism invoked for the oxidation of $n$-alkanes (up to $\mathrm{C}_{12}$ ) over molecular sieves, in which an end-on approach into small pore entrances favours terminal methyl attack. ${ }^{\mathbf{4 1}}$

The maximum TOFs for propanoic and palmitic acids are in accordance with those reported for $\mathrm{PrSO}_{3} \mathrm{H} / \mathrm{SBA}-15$ and $\mathrm{PrSO}_{3} \mathrm{H} /$ MM-SBA-15 with $340 \mathrm{~nm}$ macropores and a similar acid loading. ${ }^{21,26}$ The influence of macropore diameter on

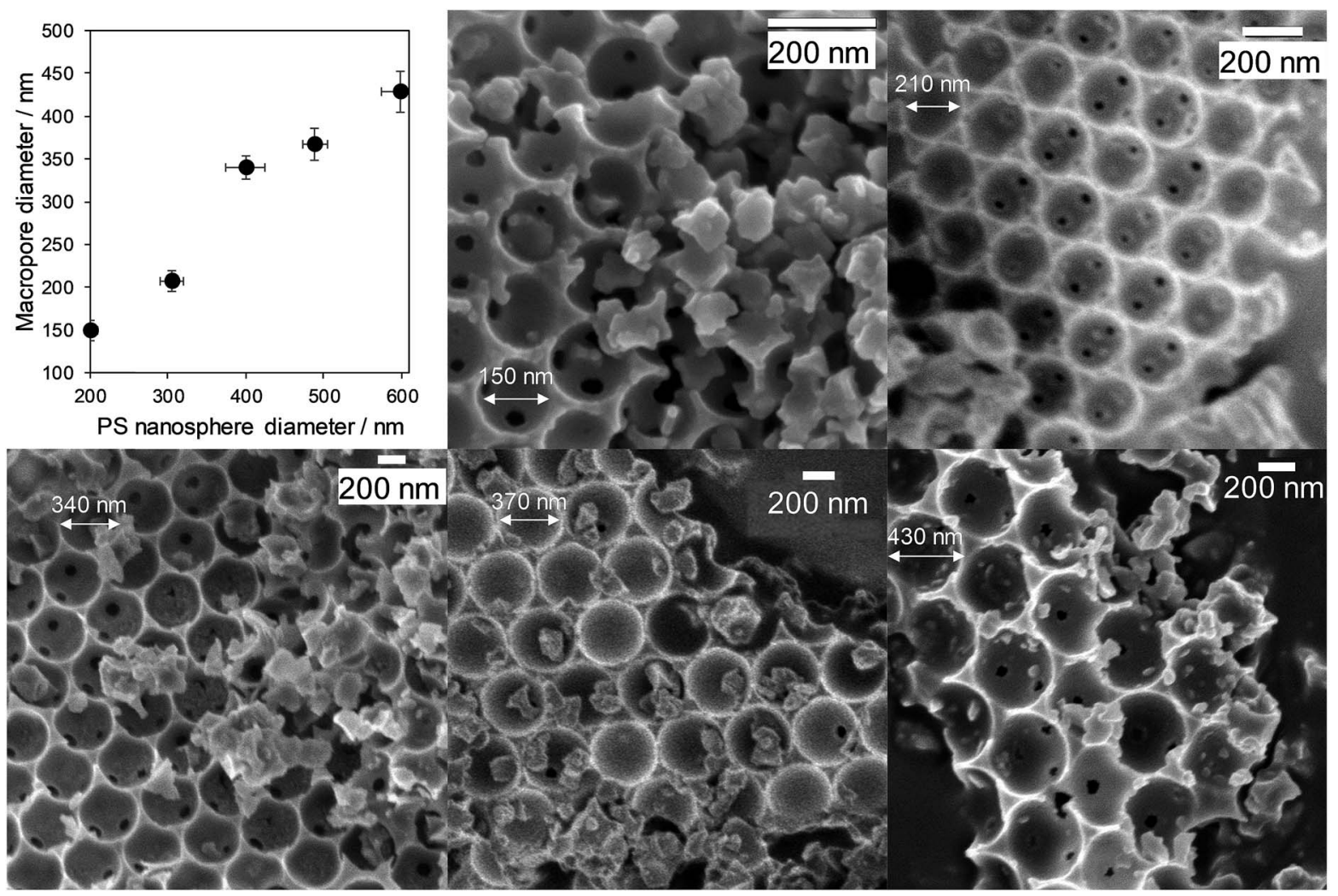

Fig. 2 Hierarchically porous SBA-15 frameworks of tunable macropore diameter: relationship between macropore and polymer template diameters, and representative SEM images. 


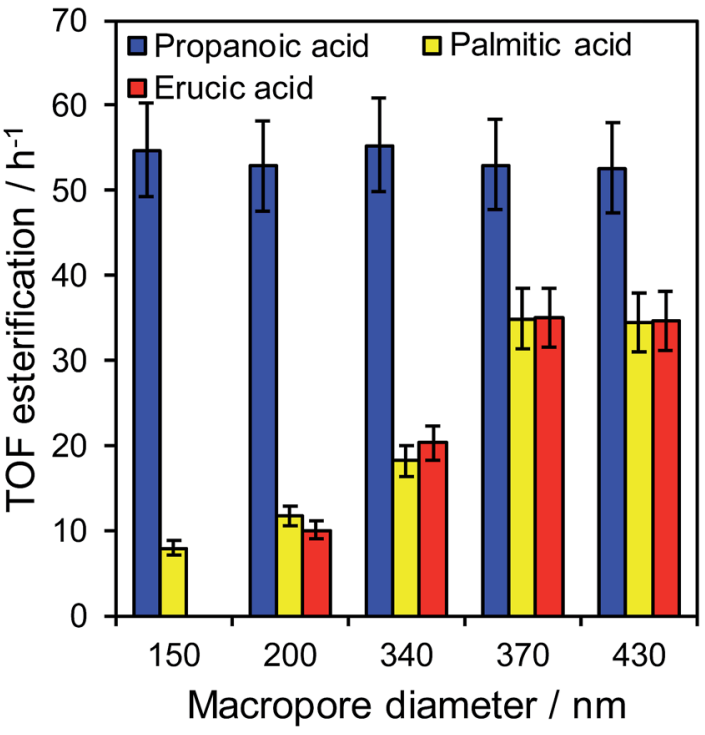

Fig. 3 Turnover frequencies for propanoic acid, palmitic acid, and erucic acid esterification over $\mathrm{PrSO}_{3} \mathrm{H} / \mathrm{MM}-\mathrm{SBA}-15$ as a function of macropore diameter.

esterification activity is solely attributable to internal pore architecture, since particle size distributions across the hierarchical catalysts were essentially constant (modal values $\sim 12 \mu \mathrm{m}$, Fig. S7†). This sensitivity of TOF to macropore and molecular size suggests that in-pore diffusion may be rate-limiting for bulky reactants; indeed the linear relationship between palmitic

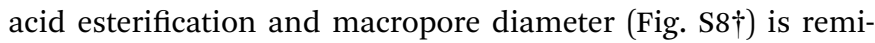
niscent of that for anticipated for Knudsen diffusion through porous media. Direct evidence of in-pore transport limitations for palmitic acid esterification was obtained from mechanical grinding of the $210 \mathrm{~nm}$ macropore catalyst. This reduced the mean particle size (and implicitly diffusion pathlength) approximately 3-fold without changing the porosity (Fig. S9 and Table S2 $\dagger$ ), resulting in a corresponding two-fold increase in TOF from 12 to $22 \mathrm{~h}^{-1}$. Apparent activation energies for palmitic acid esterification over the 210 and $430 \mathrm{~nm}$ macropore hierarchical catalysts also indicate a switchover from diffusion limited to reaction-rate limited esterification with increasing macropore size, rising from $34 \pm 3 \mathrm{~kJ} \mathrm{~mol}^{-1}(210 \mathrm{~nm})$ to $45 \pm$ $3 \mathrm{~kJ} \mathrm{~mol}^{-1}(430 \mathrm{~nm})$. The latter barrier is in fair agreement with the homogeneous acid catalysed value of $56 \mathrm{~kJ} \mathrm{~mol}^{-1}$, ${ }^{42}$ suggesting that esterification over the $430 \mathrm{~nm}$ macropore catalyst is largely free from mass transport limitations.

\section{PFG NMR diffusion studies}

Insight into the preceding dependence of TOF on catalyst structure and carboxylic acid size was subsequently derived by investigating the changes in pore connectivity with macropore diameter. PFG NMR experiments are uniquely suited to such studies, enabling the direct and non-invasive evaluation of molecular self-diffusion coefficients within liquid-saturated catalyst structures. ${ }^{43}$ For long observation times $\Delta$, the effective diffusion coefficient of the restricted liquid $D_{\text {eff }}$ is reduced from that of the unrestricted liquid $D_{0}$ (in the bulk media) by the tortuosity of the porous network $\tau$. This parameter defines the overall interconnectivity of accessible pore structures and may be estimated from: ${ }^{31}$

$$
\tau=\frac{D_{0}}{D_{\text {eff }}(\Delta \rightarrow \infty)}
$$

Here $D_{\text {eff }}(\Delta \rightarrow \infty)$ is the effective self-diffusion coefficient of the restricted liquid observed in the long-time diffusion limit, such that $D_{\text {eff }}$ is invariant to further increases in $\Delta$. Such measurements probe average molecular displacements (or root mean square displacements, RSMD $=\sqrt{2 D_{\text {eff }} \Delta}$ ), which are significantly greater than the modal pore sizes present. Eqn (1) holds for small, non-viscous, weakly-interacting liquids, and has been used to quantify the tortuosity of various mesoporous catalysts. ${ }^{4-48}$ For liquids exhibiting non-negligible interactions with the porous matrix, $\tau$ will vary with molecular structure and functionality, giving an effective tortuosity factor that may no longer represent the pore connectivity. ${ }^{28,46,48}$

In the present work, the diffusivities of cyclohexane and dodecane were explored for the preceding $150 \mathrm{~nm}$ and $430 \mathrm{~nm}$ macroporous sulfonic acid silicas. The significant difference in molecular size between these probe molecules is an approximate analogue for the structural differences between the shortand long-chain carboxylic acids in Fig. 3. The use of alkanes was necessitated by the relatively high melting points of erucic and palmitic acids $\left(33.8{ }^{\circ} \mathrm{C}\right.$ and $62.9{ }^{\circ} \mathrm{C}$ respectively) which prohibited self-diffusion measurements using our diffusion probe. Solubilising the acids in methanol is problematic due to: (i) competitive in-pore diffusion between methanol and the acid (diffusion of 2-component systems depends on their molar fraction); (ii) strong hydrogen bonding networks throughout the imbibed methanol/acid mixture and with the pore surfaces, which prevent determination of the true tortuosity of the porous catalyst (only possible when probe molecules do not chemically interact with pore surfaces or each other); and (iii) catalytic reaction resulting in changing molecule populations which is not congruent to obtaining useful diffusion information. ${ }^{46}$ Ideal probe molecules should therefore be chemically inert, free of polar functional groups, exhibit sufficiently high diffusivities that the long-time diffusion limit can be accessed (wherein probe molecules can access multiple pores during a sensible observation time of $\sim 100 \mathrm{~ms}$ ), and express long relaxation times; criteria which favour relatively 'small' probe molecules, with dodecane the largest such probe typically investigated.

Self-diffusion coefficients $D_{0}$ and $D_{\text {eff }}(\Delta \rightarrow \infty)$ are readily obtained through appropriate PFG NMR diffusion experiments. ${ }^{31}$ Log-attenuation plots for unrestricted liquids were obtained using the PGSTE pulse sequence (Fig. 4); $D_{0}$ was determined by fitting the acquired data points to the StejskalTanner equation: $:^{35}$

$$
\frac{S(g)}{S_{0}}=\exp \left\{-b D_{0}\right\}
$$

Here $S_{0}$ is the NMR signal in the absence of any applied field gradient and $S(g)$ is the acquired signal in the presence of magnetic field gradients of magnitude $g$. The $b$-factor for the PGSTE pulse sequence is: 


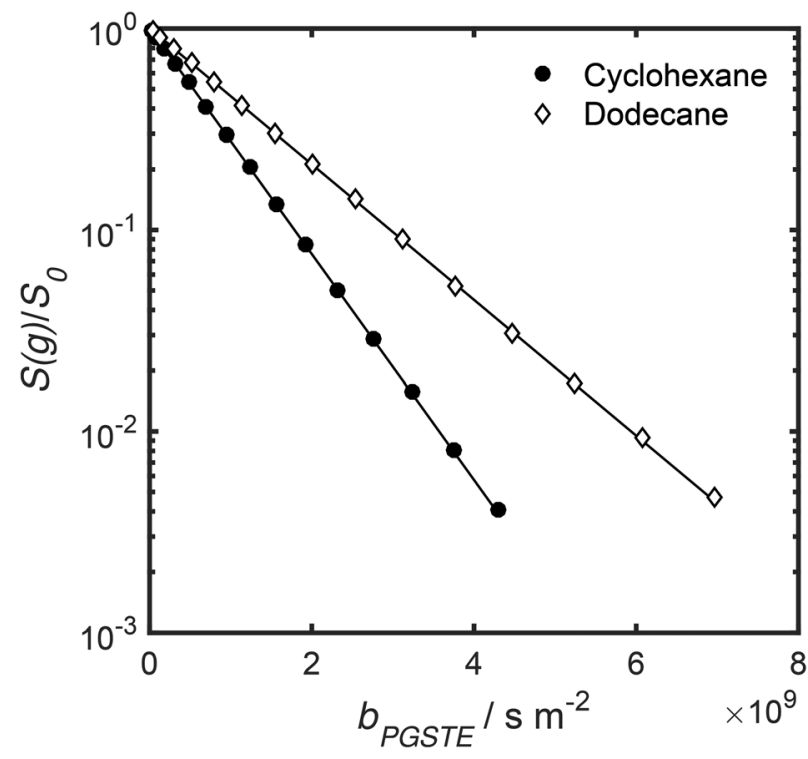

Fig. 4 PGSTE data for unrestricted cyclohexane and dodecane selfdiffusion. Solid lines are a fit to eqn (2) and (3).

$$
b_{\mathrm{PGSTE}}=\gamma^{2} g^{2} \delta^{2}\left(\Delta-\frac{\delta}{3}\right)
$$

where $\gamma$ is the gyromagnetic ratio of ${ }^{1} \mathrm{H}$ and $\delta$ is the effective gradient pulse length in Fig. 1. $D_{0}$ values for unrestricted liquids were obtained by non-linear least squares fitting of the PGSTE data according to eqn (2) and (3), and were $1.291 \pm 0.002 \times 10^{-9}$ $\mathrm{m}^{2} \mathrm{~s}^{-1}$ and $7.76 \pm 0.01 \times 10^{-10} \mathrm{~m}^{2} \mathrm{~s}^{-1}$ for cyclohexane and dodecane respectively, in excellent agreement with literature values. ${ }^{49}$

Log-attenuation data for the self-diffusion of cyclohexane and dodecane when restricted within $\mathrm{PrSO}_{3} \mathrm{H} / \mathrm{MM}-\mathrm{SBA}-15$ catalysts possessing $150 \mathrm{~nm}$ and $430 \mathrm{~nm}$ macropores were subsequently obtained (Fig. 5); these materials were selected as limiting small and large pore solid acid catalysts. The APGSTE data points were acquired using four different observation times ranging from $25-200 \mathrm{~ms}$. It is apparent that these signal attenuations do not follow a simple exponential decay of the form described in eqn (2). Indeed, the attenuation data exhibit significant curvature on a log-scale, which is qualitatively independent of the observation time over the range of $\Delta$ values explored. Extracting physically meaningful diffusion coefficients from these data requires careful consideration of the origin of this complex signal. Curved log-attenuation data are typically attributed to multicomponent exponential behaviour of the form $S(g) / S_{0}=\sum_{i} p_{i} \exp \left\{-b D_{i}\right\}$. Here, the diffusion coefficients $D_{i}$ describe the mobility of different molecular ensembles $i$ with effective populations $p_{i}$. Such an approach is generally valid if the mean lifetime of molecules within each population exceeds the observation time over which diffusion is measured (the so-called slow exchange condition), ${ }^{31}$ and if appropriate molecular environments exhibiting different length scales and connectivity exist throughout the sample to generate multiple diffusion coefficients. Normal (or Fickian) self- diffusion must be assumed within each environment, such that a Gaussian distribution of molecular displacements evolves over time. ${ }^{43,50}$ Typical examples include the long- and shortrange behaviour of gases and volatile liquids imbibed in microporous crystalline solids, wherein different diffusion coefficients may be assigned to inter- and intracrystalline diffusion. ${ }^{51}$ Differentiation between these transport phenomena primarily depends on the RMSD of the diffusing probe molecules, and hence the observation times and experimental temperatures employed. Relevant studies in which multicomponent exponential data fittings of this form have been applied include investigations of diffusion within hierarchical meso/ microporous zeolites ${ }^{52-55}$ and aggregates of mesoporous SBA$15 .{ }^{56}$ Galarneau et al. examined $n$-hexane diffusion in zeolite FAU-Y and mesoporous Al-MCM- $41,{ }^{57}$ demonstrating that selfdiffusion within a mechanical mixture of these materials could be described as a simple superposition of the diffusivities within the individual materials, whereas the self-diffusion coefficient from a hierarchical combination of these materials lay in an intermediate regime between that characteristic of mesopores or micropores. Furthermore, Adem et al. investigated the self-diffusion of $n$-hexane during the pseudomorphic synthesis of MCM- $41^{58}$ ) to explore time-dependent pore structures. Multicomponent exponential fittings distinguished between long-range interparticle displacement and intraparticle displacement within the resulting pore structures, revealing a notable dependence of the intraparticle effective self-diffusion coefficient on both pore size and connectivity.

A biexponential fitting of the form $S(g) / S_{0}=p_{\text {fast }} \exp \left\{-b D_{\text {fast }}\right\}$ $+p_{\text {slow }} \exp \left\{-b D_{\text {slow }}\right\}$ provided good agreement with our acquired attenuation data (Fig. S10 $\dagger$ ), where $D_{\text {fast }}$ describes the initial, steep signal decay at low $b$, while $D_{\text {slow }}$ describes the limiting gradient of the shallow decay observed at high $b$. Recalling the APGSTE sequence for the observation of restricted selfdiffusion, the relevant $b$-factor is: ${ }^{37}$

$$
b_{\text {APGSTE }}=\gamma^{2} g^{2} \delta^{2}\left(\Delta-\frac{\delta}{12}-\frac{\tau_{\mathrm{e}}}{2}\right)
$$

where $\tau_{\mathrm{e}}$ is the spin echo time defined in Fig. 1 . For cyclohexanesaturated materials we obtain $D_{\text {fast }} \approx 2.6 \times 10^{-10} \mathrm{~m}^{2} \mathrm{~s}^{-1}$ and $D_{\text {fast }} \approx 4.0 \times 10^{-10} \mathrm{~m}^{2} \mathrm{~s}^{-1}$ for catalysts exhibiting $150 \mathrm{~nm}$ and $430 \mathrm{~nm}$ macropores respectively (Table S3†), with associated maximum RMSDs $(\Delta=200 \mathrm{~ms})$ of 10 and $13 \mu \mathrm{m}$ probed by our cyclohexane diffusion experiments respectively. Corresponding values for dodecane are $D_{\text {fast }} \approx 1.4 \times 10^{-10} \mathrm{~m}^{2} \mathrm{~s}^{-1}$ and $D_{\text {fast }} \approx$ $2.2 \times 10^{-10} \mathrm{~m}^{2} \mathrm{~s}^{-1}$ for the two catalysts (Table S3†), with maximum RMSDs $(\Delta=200 \mathrm{~ms})$ of 7 and $9 \mu \mathrm{m}$ probed respectively. These RMSD are adequate for probing intraparticle diffusion in the present materials as our PFG NMR measurements will be dominated by diffusion through the largest catalyst particles (Fig. S7 $\uparrow$ ); the significant reduction in $D_{\text {fast }}$ (relative to $D_{0}$ ) further suggests that any spin population outside of the catalyst particles is small. The corresponding signal intensity $p_{\text {fast }}$ comprises over $95 \%$ of the acquired NMR signal in each case, such that $D_{\text {fast }}$ may be assigned to bulk-pore diffusion throughout the material. It is noteworthy that these RMSD values significantly exceed the macropore domains 

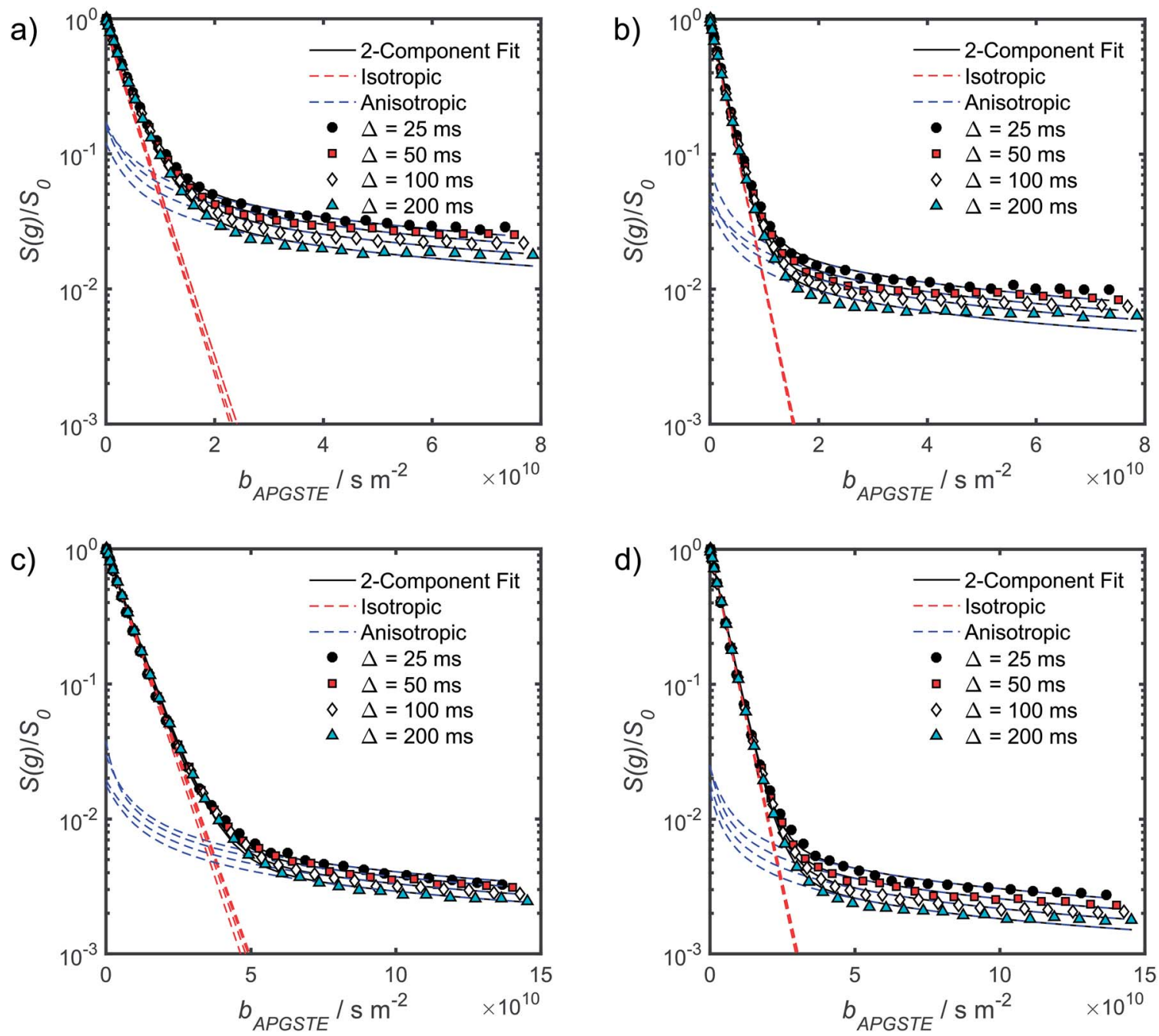

Fig. 5 APGSTE data for $\mathrm{PrSO}_{3} \mathrm{H} / \mathrm{MM}$-SBA-15 catalysts: PFG NMR signal attenuation curves for (a and b) cyclohexane saturated and (c and d) dodecane-saturated $\mathrm{PrSO}_{3} \mathrm{H} / \mathrm{MM}$-SBA-15 catalysts with respective $150 \mathrm{~nm}$ and $430 \mathrm{~nm}$ macropores. Two-component fits to the acquired data points utilised eqn (6); isotropic and anisotropic fitted components are shown in red and blue respectively.

present in either material, suggesting that $D_{\text {fast }}$ characterises diffusion occurring through both the macropores and mesopores. This postulate is supported by chemical cascade reactions using the same macroporous-mesoporous SBA-15 framework, ${ }^{34}$ wherein a high degree of connectivity between mesopores and macropores was evidenced.

The slowly decaying signal attenuation at high $b$ in Fig. 5 is more difficult to assign. Biexponential fitting yields $D_{\text {slow }} \sim$ $10^{-12} \mathrm{~m}^{2} \mathrm{~s}^{-1}$ for both the cyclohexane- and dodecane-saturated catalysts. Considering the above discussion of RMSDs and the assignment of $D_{\text {fast }}$ to diffusion through the entire pore network, it is rather surprising to identify a small spin population exhibiting such a significantly different diffusion coefficient. Such an observation might imply the existence of configurational diffusion, where liquid mobility is dominated by interactions with the pore walls within one of the domains. ${ }^{43}$ However, in the present materials mesopore diameters are too large for configurational diffusion to occur, and no micropore population was identified by $\mathrm{N}_{2}$ porosimetry $t$-plot analysis (data not shown), suggesting this slow component cannot be attributed to configurational diffusion through structural micropores. Previous studies of self-diffusion within an adsorbed surface layer reported similar PFG NMR attenuation curves to the present data. ${ }^{59,60}$ However, covalent modification of the surface with alkyl groups removed this component from the attenuation data. ${ }^{60}$ The existence of a slowly diffusing surface component can therefore be discounted in this work due to presence of surface propyl sulfonic acid groups. Furthermore, the aforementioned studies required thousands of repeat scans to observe the slowly diffusing surface layer, ${ }^{59,60}$ and hence such a population is unlikely to be detected by the limited repeat scans in this work. It is pertinent then to consider the isotropy of the pore structures under study. Kärger and co-workers have demonstrated that diffusion through mesoporous SBA-15 and MCM-41 silicas may result in curved PFG log-attenuation plots due to their anisotropic pore structure. ${ }^{61-63}$ For a powderaverage of saturated anisotropic pores, the PFG signal is expected to attenuate according to: ${ }^{63}$ 


$$
\frac{S(g)}{S_{0}}=\frac{\sqrt{\pi}}{2} \frac{\exp \left\{-b D_{\text {perp }}\right\} \operatorname{erf}\left\{\sqrt{b\left(D_{\text {par }}-D_{\text {perp }}\right)}\right\}}{\sqrt{b\left(D_{\text {par }}-D_{\text {perp }}\right)}}
$$

where $D_{\text {par }}$ and $D_{\text {perp }}$ are self-diffusion coefficients describing the rates of displacement parallel and perpendicular to the direction of the mesoporous channels, respectively, and $\operatorname{erf}(\cdots)$ is the error function. Recalling that the hierarchical structure of the materials explored here comprises both isotropic macropores and anisotropic mesopores, we suggest an appropriate attenuation equation will be of the form

$$
\begin{aligned}
\frac{S(g)}{S_{0} \approx} & p_{\text {iso }} \exp \left\{-b D_{\text {iso }}\right\} \\
& +p_{\text {aniso }} \frac{\sqrt{\pi}}{2} \frac{\exp \left\{-b D_{\text {perp }}\right\} \operatorname{erf}\left\{\sqrt{b\left(D_{\text {par }}-D_{\text {perp }}\right)}\right\}}{\sqrt{b\left(D_{\text {par }}-D_{\text {perp }}\right)}}
\end{aligned}
$$

Similar expressions have been used to quantify self-diffusion within SBA-15 aggregates ${ }^{56}$ and aluminosilicate nanotubes. ${ }^{64,65}$ Here, $D_{\text {iso }}$ is an isotropic diffusion coefficient similar to $D_{\text {fast }}$ described above; we propose that $D_{\text {iso }}$ represents diffusion throughout the entire catalyst, and is dominated by the isotropic macropores. The signal fractions $p_{\text {iso }}$ and $p_{\text {aniso }}$ describe the magnitude of attenuated signals due to the isotropic and anisotropic components of eqn (6) respectively, and in the following fitting processes have been set such that $p_{\text {iso }}=1-p_{\text {aniso. }}$. We propose that $p_{\text {aniso }}$ represents a small spin population which remains within mesoporous channels for the entire observation time $\Delta$, and which attenuates according to eqn (5), while $p_{\text {iso }}$ represents the remainder of the spin ensemble.

It is appropriate here to highlight the recent development by Splith et al. ${ }^{66}$ of a complex model to address diffusive exchange between isotropic and anisotropic environments. The authors considered the diffusion and exchange of water within and between crystals of the anisotropic microporous metal-organicframework Al fumarate. The resulting model improved fitting of PFG echo attenuation data relative to that achieved using eqn (5) but required that population of the isotropic environment was negligible. Such an approximation is appropriate for systems containing saturated anisotropic materials with only vapour phase within the intercrystalline voids, but is clearly not valid for our hierarchical catalysts which comprise fullysaturated isotropic and anisotropic pores. A different approach was therefore adopted in which APGSTE data for liquid-saturated $\mathrm{PrSO}_{3} \mathrm{H} / \mathrm{MM}$-SBA-15 pellets were fit to eqn (4) and (6) using a non-linear least squares fitting. The resulting two-component fit is in good agreement with the acquired data; representative curves are shown in Fig. 5, where the isotropic and anisotropic contributions of eqn (6) are deconvoluted for clarity. This deconvolution visualises the slowly decaying signal at high $b$ resulting from a small spin population exhibiting anisotropic diffusion. A small decrease in the signal obtained from this population ( $p_{\text {aniso }}$ ) is evident with increasing $\Delta$. There are two possible explanations for this decrease: (i) nuclear spin $T_{1}$ relaxation during the longitudinal storage period $\mathrm{T}=\Delta-$ $2 \tau_{\mathrm{e}}$, facilitating additional signal attenuation with increasing $\Delta$; (ii) diffusive exchange, reducing the number of spins experiencing purely anisotropic self-diffusion during $\Delta$. Since longitudinal relaxation time constants $T_{1}$ (Table S4 $\dagger$ ) were at least an order of magnitude greater than the maximum $\Delta$ value employed, molecular exchange appears the dominant mechanism underpinning the decrease in $p_{\text {aniso. }}$ A decrease in $p_{\text {aniso }}$ with increasing macropore size is also apparent, as would be expected from the concomitant increase in $p_{\text {iso. The inherent }}$ differences in $p_{\text {aniso }}$ for the two probe liquids may be rationalised by considering the enhanced $T_{1}$ relaxation rates $\left(1 / T_{1}\right)$ exhibited by the dodecane-versus cyclohexane saturated catalysts (Table S4†).

Unfortunately, the fits in Fig. 5 are of insufficient quality to robustly estimate $D_{\text {perp }}$; we attribute this to dominance of the acquired data by isotropic signal attenuation at small $b$, and hence focus discussion on $D_{\text {iso }}$ and $D_{\mathrm{par}}$. These diffusion coefficients (Table $55 \dagger$ ) are shown as a function of observation time in Fig. 6, and are of equivalent orders of magnitude $\left(\sim 10^{-10} \mathrm{~m}^{2} \mathrm{~s}^{-1}\right)$ in contrast to the diffusion coefficients assigned through the preceding biexponential fitting (Table S3†). Self-diffusion coefficients obtained from the isotropic component of eqn (6) appear in Fig. 6a. For the dodecane-saturated catalysts, and cyclohexane-saturated analogue with $150 \mathrm{~nm}$ macropores, a small decrease in $D_{\text {iso }}$ occurs with increasing $\Delta$ over the range $25-100 \mathrm{~ms}$. Since the observed $D_{\text {iso }}$ values are all significantly reduced from $D_{0}$, we interpret these diffusivities as indicative of an intermediate regime between unrestricted and restricted diffusion. ${ }^{31}$ Molecules therefore experience significant interactions with the pore walls but do not displace through a large enough sample of the pore space to be considered within the long-time diffusion limit. Above $\Delta=100 \mathrm{~ms} D_{\text {iso }}$ is invariant, with dodecane presenting asymptotic $D_{\text {iso }}(\Delta \rightarrow \infty) \cong D_{\text {iso }}(\Delta=100 \mathrm{~ms})$ values of $\sim 1.37 \times 10^{-10} \mathrm{~m}^{2} \mathrm{~s}^{-1}$ and $\sim 2.27 \times 10^{-10} \mathrm{~m}^{2} \mathrm{~s}^{-1}$ within the $150 \mathrm{~nm}$ and $430 \mathrm{~nm}$ macropore catalysts respectively, and cyclohexane presenting an asymptotic value of $\sim 2.86 \times 10^{-10}$ $\mathrm{m}^{2} \mathrm{~s}^{-1}$ within the $150 \mathrm{~nm}$ macropore catalyst. These diffusivities may therefore be interpreted as a probe of pore connectivity. It is interesting to note that we do not observe the same trend when considering cyclohexane-saturated $\mathrm{PrSO}_{3} \mathrm{H} / \mathrm{MM}-\mathrm{SBA}-15$ with $430 \mathrm{~nm}$ macropores, which exhibits by far the largest $D_{\text {iso }}$ of the alkane/silica systems investigated (measurements were conducted multiple times to confirm this anomaly). In this latter case, a slight but steady increase in $D_{\text {iso }}$ with increasing $\Delta$ was observed suggesting that $D_{\text {iso }}$ observed at small $\Delta$ is already indicative of the long-time diffusion limit. The small increase in $D_{\text {iso }}$ with increasing $\Delta$ suggests an increased propensity for the imbibed cyclohexane to diffuse out of the particles and into the intercrystalline voids during large observation times. For the purposes of assigning a long-time diffusion limit value to this system we take a $D_{\text {iso }}(\Delta \rightarrow \infty) \cong D_{\text {iso }}(\Delta=25 \mathrm{~ms})$ value of $\sim 4.35 \times 10^{-10} \mathrm{~m}^{2} \mathrm{~s}^{-1}$.

Values of the anisotropic diffusion coefficient $D_{\text {par }}$ are presented in Fig. 6b. Despite greater uncertainty in these $D_{\text {par }}$ values, fitting clearly reveals that $D_{\mathrm{par}}>D_{\text {iso }}$, suggesting diffusion is faster through the mesopore network than the overall hierarchical pore structure. This finding is in agreement with 

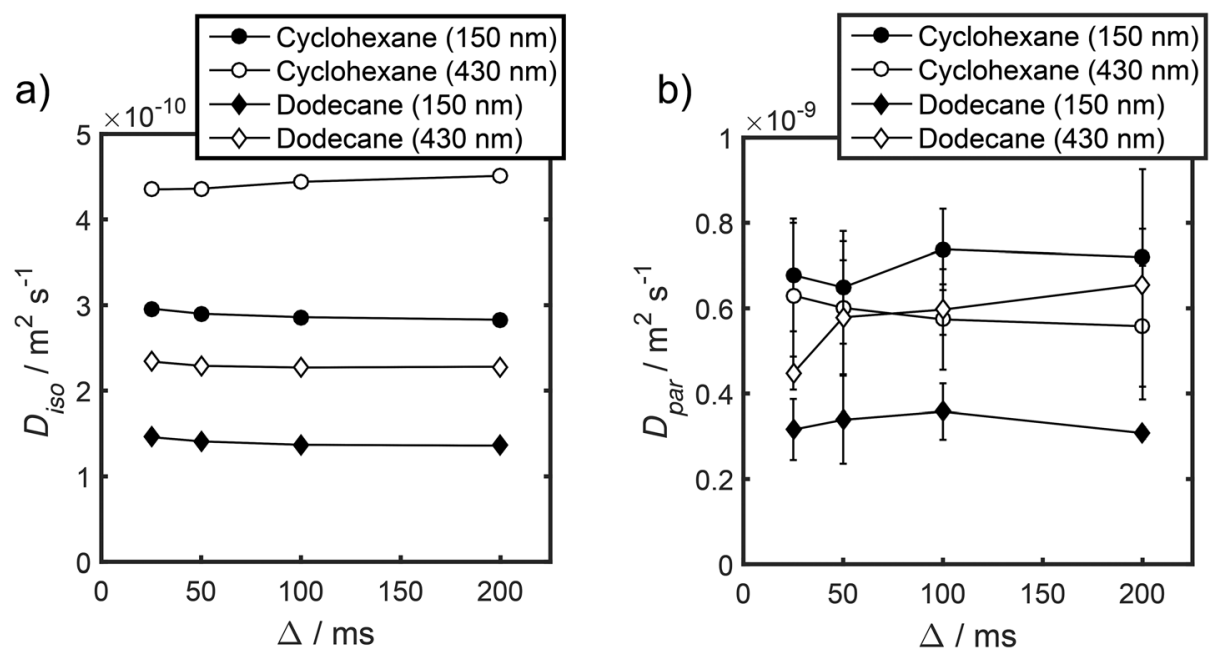

Fig. 6 (a) $D_{\text {iso }}$ and (b) $D_{\text {par }}$ values obtained by fitting APGSTE data to eqn (6). Macropore diameters are indicated in parentheses. Errors represent \pm 1 standard deviation of three measurements of three different samples and are too small to be observed in (a).

Adem et al., who reported a distinct decrease in the effective self-diffusivity of $n$-hexane imbibed within MCM- 41 upon the addition of large, secondary mesopores embedded in the structure which create 'stagnation zones'. ${ }^{58}$ More detailed insight is obtained from the ratios $D_{0} / D_{\text {iso }}$ and $D_{0} / D_{\text {par }}$ (Table S6†), which we interpret as providing direct insight into pore connectivity within the catalyst structures studied. In particular, $D_{0} / D_{\text {iso }}$ reflects the pore connectivity of the overall catalyst structure, while $D_{0} / D_{\text {par }}$ reflects that of the anisotropic mesopores. These ratios are plotted as a function of observation time in Fig. 7. The ratio $D_{0} / D_{\text {iso }}$ is shown in Fig. 7a and b for each catalyst/alkane combination, and is invariant at $\Delta \geq 100$ ms, consistent with the preceding analysis of their effective self-diffusion coefficients. These values are therefore considered indicative of the long-time diffusion limit, and hence a probe of pore connectivity. The anomalous behaviour of cyclohexane within $\mathrm{PrSO}_{3} \mathrm{H} / \mathrm{MM}-\mathrm{SBA}-15$ with $430 \mathrm{~nm}$ macropores is no longer apparent when interpreted as a $D_{0} / D_{\text {iso }}$ ratio and is essentially invariant to increasing observation time
(Fig. 7a). Values of this ratio for the four alkane/catalyst systems are summarised in Table 2 and reveal two notable features. First, $D_{0} / D_{\text {iso }}$ is greater for the catalyst with $150 \mathrm{~nm}$ macropores than that with $430 \mathrm{~nm}$ macropores, irrespective of the probe molecule considered, i.e. overall pore network tortuosity decreases with increasing macropore size. Second, $D_{0} / D_{\text {iso }}$ values for cyclohexane and dodecane are significantly different for the same macropore size; dodecane values are approximately $20 \%$ larger for both macropore sizes. A consequence of the latter is that these values represent an effective tortuosity which is influenced by molecular size and/shape. However, since the change in $D_{0} / D_{\text {iso }}$ with macropore size is qualitatively independent of the probe liquid, we can remain confident that an increase in macropore size is indeed associated with a decrease in overall tortuosity.

Fig. 7b also shows $D_{0} / D_{\text {par }}$ as a function of observation time. The uncertainty in these values is significant, and stems from errors in assigning $D_{\text {par }}$ during fitting (Table S5 $\dagger$ ). Nevertheless, there is clearly substantial overlap between the four datasets,
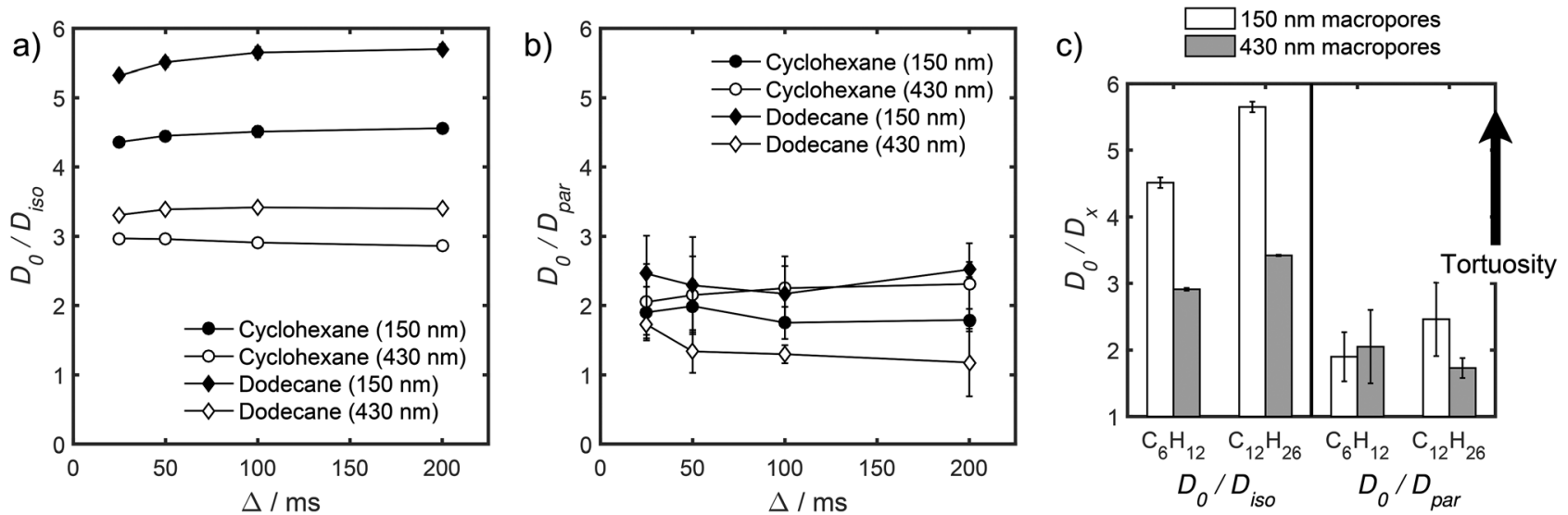

Fig. 7 (a and b) Effective tortuosity parameters as a function of observation time, and (c) comparative summary for PrSO 3 H/MM-SBA-15 with different macropores and probe molecules ( $\Delta=100 \mathrm{~ms}$ for $D_{0} / D_{\text {iso }}$ values and $\Delta=25 \mathrm{~ms}$ for $D_{0} / D_{\text {par }}$ values). 
Table 2 Diffusion parameters for $\mathrm{PrSO}_{3} \mathrm{H} / \mathrm{MM}-\mathrm{SBA}-15$ materials in the long-time diffusion limit

\begin{tabular}{llclr}
\hline Probe molecule & Macropore diameter $/ \mathrm{nm}$ & $D_{0} / 10^{-10} \mathrm{~m}^{2} \mathrm{~s}^{-1}$ & $D_{\text {iso }}(\Delta \rightarrow \infty) / 10^{-10} \mathrm{~m}^{2} \mathrm{~s}^{-1}$ & $D_{0} / D_{\text {iso }}(\Delta \rightarrow \infty)$ \\
\hline Cyclohexane & 150 & $12.91 \pm 0.02$ & $2.86 \pm 0.05$ & $4.51 \pm 0.08$ \\
Dodecane & 430 & & $4.35 \pm 0.02$ & $2.97 \pm 0.01$ \\
& 150 & $7.76 \pm 0.01$ & $1.37 \pm 0.02$ & $5.65 \pm 0.08$ \\
& 430 & & $2.27 \pm 0.01$ & $3.42 \pm 0.02$
\end{tabular}

particularly clear at short observation times, where the RMSD is significantly less than the modal catalyst particle size (Table S5†). This overlap strongly suggests that the interconnectivity (and pore diameter and curvature) of the mesopores is similar in both catalysts, as expected given that the only difference in their syntheses was the size of sacrificial polymeric bead used to form the macropores, and further evidences that the tortuosity of our $\mathrm{PrSO}_{3} \mathrm{H} / \mathrm{MM}$-SBA-15 catalysts is solely controlled by macropore size. General agreement (within experimental error)

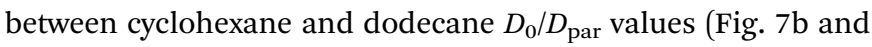
c) indicates that diffusive displacement within the mesopores of both catalysts is largely independent of molecular size/shape.

\section{Structure-reactivity correlations}

We first note that macropores were varied independently of the size or tortuosity of mesopores (Fig. S5, S6† and 7c), enabling the influence of macropores on carboxylic acid esterification by the hierarchical catalysts to be rationalised. For propanoic acid, esterification was independent of macropore size, as may be anticipated from its small kinetic diameter $(\sim 0.48 \mathrm{~nm})$ and hence minimal diffusive restriction by the pore network. A recent PFG NMR study on organic acid diffusion through mesoporous SBA-15 and KIT-6 silicas ${ }^{47}$ reported that the effective tortuosity increased with acid chain length, and that pore walls enhanced diffusion possibly by disrupting the dynamic hydrogen bonding network present throughout the liquid mixture (as has previously observed for polyols and polyol/ methanol mixtures ${ }^{\mathbf{4 1 - 4 4}}$ ). It follows that propanoic acid selfdiffusion may be dominated by hydrogen-bonding dynamics, rather than by pore network tortuosity, consistent with the present findings that propanoic acid TOF and $D_{0} / D_{\text {iso }}$ (equated here to macropore size) are uncorrelated. Conversely, the preceding SBA-15 and KIT-6 PFG-NMR study showed that longchain acid diffusion was dominated by (meso)pore tortuosity and molecular size. ${ }^{45}$ We might therefore expect the TOFs for palmitic and erucic acids to follow the tortuosity of our hierarchical $\mathrm{PrSO}_{3} \mathrm{H} / \mathrm{MM}-\mathrm{SBA}-15$ catalysts, as indeed is observed; long-chain acids TOFs are inversely correlated with $D_{0} / D_{\text {iso }}$.

PFG NMR analysis of the diffusion of solvated acids was not possible due to competing catalytic esterification. It is therefore insightful to consider the difference in effective tortuosity experienced by cyclohexane and dodecane within the small and large macropore catalysts (Table 2). Dodecane exhibits greater $D_{0} / D_{\text {iso }}$ values than the smaller cyclohexane probe molecule, irrespective of macropore size. It follows that a secondary, sizedependent transport resistance must be present, which further reduces the mobility of large molecules in addition to restrictions imposed by the inherent pore network tortuosity. Since dodecane lacks any polar functionality, this secondary transport resistance cannot be attributed to e.g. enhanced interactions with surface sulfonic acid groups or hydroxyls, but rather reflects topological constraints of the pore system. This finding agrees with pore-scale diffusion simulations, ${ }^{67,68}$ which reveal that the effective tortuosity of mesoporous SBA-15 and KIT-6 silicas depends on the size of the probe molecule. Note that this contrasts with simulations ${ }^{67,68}$ and PFG-NMR studies of amorphous mesoporous silicas, for which effective tortuosity is independent of molecular size. ${ }^{46}$ Relative $D_{0} / D_{\text {iso }}$ values for cyclohexane versus dodecane through $\mathrm{PrSO}_{3} \mathrm{H} / \mathrm{MM}-\mathrm{SBA}-15$ also depend on macropore size, being $23 \%$ greater for $150 \mathrm{~nm}$ macropores and $15 \%$ greater for $430 \mathrm{~nm}$ macropores (Table 2), i.e. the secondary transport resistance identified above decreases with increasing macropore size. This observation manifests our chemical intuition that larger macropores do indeed enhance mass transport of long-chain acids through hierarchically porous catalysts. In contrast $D_{0} / D_{\text {par }}$, which reflects diffusion through only the mesopores, is approximately independent of probe molecule. This suggests that the size-dependent transport resistance seen for $D_{0} / D_{\text {iso }}$ arises from: (i) topological effects at the macropore/mesopore interface, i.e. bulkier molecules (dodecane, palmitic or erucic acid) struggle to enter mesopores, but once correctly oriented can diffuse relatively freely along mesopore channels (recall that $D_{\text {par }}>D_{\text {iso) }}$ ); and/or (ii) stagnation zones created by small macropores that are embedded in the silica framework and hinder transport through the mesopore network. Constricted pore openings are reported for mesoporous MCM-41 and SBA-15.67,69 A detailed analysis of secondary transport resistances within these hierarchically porous catalysts is the subject of future studies.

\section{Conclusions}

The synthesis and characterisation of hierarchical macroporous-mesoporous SBA-15 sulfonic acid catalysts exhibiting tunable macropore sizes is reported, facilitating new insight into transport phenomena through hierarchically porous materials. Catalytic performance of the resulting $\mathrm{PrSO}_{3} \mathrm{H} / \mathrm{MM}$ SBA-15 family towards the liquid phase esterification of short and long-chain carboxylic acids with methanol revealed that turnover frequencies for propanoic acid were structure invariant, whereas those for palmitic and erucic acids exhibit striking increases with macropore size. Complementary PFG NMR diffusion measurements enable the non-invasive assessment of pore connectivity and diffusive restrictions for liquids 
imbibed within these catalysts. Effective self-diffusion of cyclohexane and dodecane within $\mathrm{PrSO}_{3} \mathrm{H} / \mathrm{MM}-\mathrm{SBA}-15$ catalysts containing $150 \mathrm{~nm}$ and $430 \mathrm{~nm}$ macropores reveals complex signal attenuation data, attributed to a superposition of isotropic and anisotropic contributions. Diffusivities extracted from these measurements provide an estimate of the structural tortuosity through comparison with bulk selfdiffusion coefficients. Tortuosity of the overall pore network decreases with increasing macropore size, indicating that larger macropores improve pore connectivity throughout the hierarchical framework. Larger macropores enhance longchain carboxylic acid esterification by improving pore structure connectivity and hence active site accessibility. Propanoic acid esterification is indifferent to the tortuosity of our hierarchically porous catalysts, consistent with the diffusive behaviour of short-chain carboxylic acids in $\mathrm{SBA}-15,{ }^{47}$ and presumably reflecting its small kinetic diameter. Molecular size is an important factor in determining the extent of diffusive restriction imposed by the hierarchical pore networks, and increased for larger probe molecules resulting in larger effective tortuosities for dodecane than cyclohexane. This additional restriction was more pronounced for smaller macropores exacerbating the poor catalytic turnover for palmitic and erucic acids within small (150 nm) macropore $\mathrm{PrSO}_{3} \mathrm{H} / \mathrm{MM}-\mathrm{SBA}-15$ catalysts.

In summary, PFG-NMR analysis shows that diffusion limitations are not related to a single textural parameter, but rather depend on a convolution of molecular size, pore connectivity, and pore diameter. Pore size and connectivity are themselves not mutually exclusive, since we have shown that altering the macropore diameter also change the effective tortuosity throughout hierarchical pore networks (a measure of connectivity). Trends in catalytic esterification are explicable by considering the enhanced mass transport attainable through tailoring macropore size (and attendant pore connectivity) to the reactant size. This work highlights the significant capability of PFG NMR experiments for elucidating pore structure and connectivity within complex porous solids, and more specifically as a powerful tool to guide the design of hierarchically porous catalysts for maximal activity.

\section{Conflicts of interest}

There are no conflicts to declare.

\section{Acknowledgements}

We would like to acknowledge the EPSRC for funding this work (grants EP/G007594/4 and EP/F063423/2). N. R. acknowledges the Catalysis@Cambridge initiative, University of Cambridge, for the award of a PhD studentship. C. D. A. would like to acknowledge Wolfson College, Cambridge, for supporting his research activities. The authors also thank Prof Lynn F. Gladden, University of Cambridge, for access to the NMR equipment.

\section{References}

1 A. G. Slater and A. I. Cooper, Science, 2015, 348, aaa8075.

2 M. E. Davis, Nature, 2002, 417, 813.

3 X.-Y. Yang, L.-H. Chen, Y. Li, J. C. Rooke, C. Sanchez and B.-L. Su, Chem. Soc. Rev., 2017, 46, 481-558.

4 J. Kim, M. Choi and R. Ryoo, J. Catal., 2010, 269, 219-228.

5 M. Milina, S. Mitchell, P. Crivelli, D. Cooke and J. PerezRamirez, Nat. Commun., 2014, 5, 10.

6 J. A. Hunns, M. Arroyo, A. F. Lee, J. M. Escola, D. Serrano and K. Wilson, Catal. Sci. Technol., 2016, 6, 2560-2564.

7 L. Y. Jia, M. Raad, S. Hamieh, J. Toufaily, T. Hamieh, M. M. Bettahar, G. Mauviel, M. Tarrighi, L. Pinard and A. Dufour, Green Chem., 2017, 19, 5442-5459.

8 M. Linares, C. Vargas, A. Garcia, C. Ochoa-Hernandez, J. Cejka, R. A. Garcia-Munoz and D. P. Serrano, Catal. Sci. Technol., 2017, 7, 181-190.

9 J. C. Manayil, A. Osatiashtiani, A. Mendoza, C. M. A. Parlett, M. A. Isaacs, L. J. Durndell, C. Michailof, E. Heracleous, A. Lappas, A. F. Lee and K. Wilson, ChemSusChem, 2017, 10, 3506-3511.

10 C. M. Parlett, P. Keshwalla, S. G. Wainwright, D. W. Bruce, N. S. Hondow, K. Wilson and A. F. Lee, ACS Catal., 2013, 3, 2122-2129.

11 T. Witoon, S. Bumrungsalee, M. Chareonpanich and J. Limtrakul, Energy Convers. Manage., 2015, 103, 886-894.

12 J. J. Woodford, J.-P. Dacquin, K. Wilson and A. F. Lee, Energy Environ. Sci., 2012, 5, 6145-6150.

13 J. Lynch, Plant Physiol., 1995, 109, 7.

14 P. Trogadas, M. M. Nigra and M.-O. Coppens, New J. Chem., 2016, 40, 4016-4026.

15 S. Gheorghiu and M.-O. Coppens, AIChE J., 2004, 50, 812820.

16 M.-O. Coppens and G. F. Froment, Chem. Eng. Sci., 1995, 50, 1013-1026.

17 G. Wang and M.-O. Coppens, Chem. Eng. Sci., 2010, 65, 23442351.

18 P. Zeigermann, S. Naumov, S. Mascotto, J. Kärger, B. M. Smarsly and R. Valiullin, Langmuir, 2012, 28, 36213632.

19 M. A. Sadeghi, M. Aghighi, J. Barralet and J. T. Gostick, Chem. Eng. J., 2017, 330, 1002-1011.

20 F. Li, Z. Wang, N. S. Ergang, C. A. Fyfe and A. Stein, Langmuir, 2007, 23, 3996-4004.

21 J. Dhainaut, J.-P. Dacquin, A. F. Lee and K. Wilson, Green Chem., 2010, 12, 296-303.

22 D. Kuang, T. Brezesinski and B. Smarsly, J. Am. Chem. Soc., 2004, 126, 10534-10535.

23 T. Sen, G. J. T. Tiddy, J. L. Casci and M. W. Anderson, Chem. Mater., 2004, 16, 2044-2054.

24 B. H. Jones and T. P. Lodge, Chem. Mater., 2011, 23, 48244831.

25 G. Lee, E. Choi, S. Yang and E.-B. Cho, J. Phys. Chem. C, 2018, 122, 4507-4516.

26 C. Pirez, J.-M. Caderon, J.-P. Dacquin, A. F. Lee and K. Wilson, ACS Catal., 2012, 2, 1607-1614. 
27 H. Li, B. Hou, J. Wang, X. Huang, C. Chen, Z. Ma, J. Cui, L. Jia, D. Sun and D. Li, Catal. Sci. Technol., 2017, 7, 38123822.

28 C. D'Agostino, M. D. Mantle and L. F. Gladden, Microporous Mesoporous Mater., 2018, 269, 88-92.

29 C. D'Agostino, Y. Ryabenkova, P. J. Miedziak, S. H. Taylor, G. J. Hutchings, L. F. Gladden and M. D. Mantle, Catal. Sci. Technol., 2014, 4, 1313-1322.

30 M. P. Hollewand and L. F. Gladden, Chem. Eng. Sci., 1995, 50, 309-326.

31 W. S. Price, NMR studies of translational motion: principles and applications, Cambridge University Press, 2009.

32 P. T. Callaghan, Translational Dynamics and Magnetic Resonance: Principles of Pulsed Gradient Spin Echo NMR, Oxford University Press, 2011.

33 T. Sen, G. Tiddy, J. Casci and M. Anderson, Chem. Mater., 2004, 16, 2044-2054.

34 C. M. Parlett, M. A. Isaacs, S. K. Beaumont, L. M. Bingham, N. S. Hondow, K. Wilson and A. F. Lee, Nat. Mater., 2016, 15, 178-182.

35 E. O. Stejskal and J. E. Tanner, J. Chem. Phys., 1965, 42, 288292.

36 J. Mitchell, T. Chandrasekera, M. Johns, L. Gladden and E. Fordham, Phys. Rev. E: Stat., Nonlinear, Soft Matter Phys., 2010, 81, 026101.

37 R. Cotts, M. Hoch, T. Sun and J. Markert, J. Magn. Reson., 1989, 83, 252-266.

38 J. E. Tanner, J. Chem. Phys., 1970, 52, 2523-2526.

39 Y. Zhang, H. Liang, C. Y. Zhao and Y. Liu, J. Mater. Sci., 2009, 44, 931-938.

40 J.-P. Dacquin, H. E. Cross, D. R. Brown, T. Düren, J. J. Williams, A. F. Lee and K. Wilson, Green Chem., 2010, 12, 1383-1391.

41 J. M. Thomas and R. Raja, Chem. Commun., 2001, 675-687, DOI: $10.1039 / \mathrm{b} 100369 \mathrm{k}$.

42 C. Beula and P. S. T. Sai, Int. J. Chem. Eng. Appl., 2013, 4, 388392.

43 F. Stallmach and J. Kärger, Adsorption, 1999, 5, 117-133.

44 C. D'Agostino, T. Kotionova, J. Mitchell, P. J. Miedziak, D. W. Knight, S. H. Taylor, G. J. Hutchings, L. F. Gladden and M. D. Mantle, Chem.-Eur. J., 2013, 19, 11725-11732.

45 C. D'Agostino, G. L. Brett, P. J. Miedziak, D. W. Knight, G. J. Hutchings, L. F. Gladden and M. D. Mantle, Chem.Eur. J., 2012, 18, 14426-14433.

46 C. D'Agostino, J. Mitchell, L. F. Gladden and M. D. Mantle, J. Phys. Chem. C, 2012, 116, 8975-8982.

47 T. J. Rottreau, C. M. Parlett, A. F. Lee and R. Evans, J. Phys. Chem. C, 2017, 121, 16250-16256.

48 M. D. Mantle, D. I. Enache, E. Nowicka, S. P. Davies, J. K. Edwards, C. D'Agostino, D. P. Mascarenhas, L. Durham, M. Sankar and D. W. Knight, J. Phys. Chem. C, 2010, 115, 1073-1079.

49 M. Holz, S. R. Heil and A. Sacco, Phys. Chem. Chem. Phys., 2000, 2, 4740-4742.
50 F. Stallmach and P. Galvosas, Annu. Rep. NMR Spectrosc., 2007, 61, 51-131.

51 J. Kärger, D. M. Ruthven and D. N. Theodorou, Diffusion in Nanoporous Materials, 2012, pp. 347-394.

52 P. Kortunov, S. Vasenkov, J. Kärger, R. Valiullin, P. Gottschalk, M. Fé Elía, M. Perez, M. Stöcker, B. Drescher, G. McElhiney, C. Berger, R. Gläser and J. Weitkamp, J. Am. Chem. Soc., 2005, 127, 13055-13059.

53 D. Mehlhorn, R. Valiullin, J. Kärger, K. Cho and R. Ryoo, Microporous Mesoporous Mater., 2012, 164, 273-279.

54 A. Menjoge, S. A. Bradley, D. B. Galloway, J. J. Low, S. Prabhakar and S. Vasenkov, Microporous Mesoporous Mater., 2010, 135, 30-36.

55 D. Mehlhorn, A. Inayat, W. Schwieger, R. Valiullin and J. Kärger, ChemPhysChem, 2014, 15, 1681-1686.

56 A. R. Menjoge, Q. Huang, B. Nohair, M. Eic, W. Shen, R. Che, S. Kaliaguine and S. Vasenkov, J. Phys. Chem. C, 2010, 114, 16298-16308.

57 A. Galarneau, F. Guenneau, A. Gedeon, D. Mereib, J. Rodriguez, F. Fajula and B. Coasne, J. Phys. Chem. C, 2016, 120, 1562-1569.

58 Z. Adem, F. Guenneau, M.-A. Springuel-Huet, A. Gédéon, J. Iapichella, T. Cacciaguerra and A. Galarneau, J. Phys. Chem. C, 2012, 116, 13749-13759.

59 T. G. A. Youngs, D. Weber, L. F. Gladden and C. Hardacre, J. Phys. Chem. C, 2009, 113, 21342-21352.

60 D. Weber, A. J. Sederman, M. D. Mantle, J. Mitchell and L. F. Gladden, Phys. Chem. Chem. Phys., 2010, 12, 2619-2624.

61 F. Stallmach, J. Kärger, C. Krause, M. Jeschke and U. Oberhagemann, J. Am. Chem. Soc., 2000, 122, 9237-9242.

62 F. Stallmach, A. Gräser, J. Kärger, C. Krause, M. Jeschke, U. Oberhagemann and S. Spange, Microporous Mesoporous Mater., 2001, 44, 745-753.

63 S. Naumov, R. Valiullin, J. Kärger, R. Pitchumani and M.-O. Coppens, Microporous Mesoporous Mater., 2008, 110, 37-40.

64 M. Dvoyashkin, J. Zang, G. I. Yucelen, A. Katihar, S. Nair, D. S. Sholl, C. R. Bowers and S. Vasenkov, J. Phys. Chem. C, 2012, 116, 21350-21355.

65 M. Dvoyashkin, A. Wang, A. Katihar, J. Zang, G. I. Yucelen, S. Nair, D. S. Sholl, C. R. Bowers and S. Vasenkov, Microporous Mesoporous Mater., 2013, 178, 119-122.

66 T. Splith, D. Fröhlich, S. K. Henninger and F. Stallmach, J. Magn. Reson., 2018, 291, 40-46.

67 S.-J. Reich, A. Svidrytski, A. Höltzel, J. Florek, F. Kleitz, W. Wang, C. Kübel, D. Hlushkou and U. Tallarek, J. Phys. Chem. C, 2018, 122, 12350-12361.

68 S.-J. Reich, A. Svidrytski, D. Hlushkou, D. Stoeckel, C. Kübel, A. Höltzel and U. Tallarek, Ind. Eng. Chem. Res., 2018, 57, 3031-3042.

69 H. Tüysüz, C. W. Lehmann, H. Bongard, B. Tesche, R. Schmidt and F. Schüth, J. Am. Chem. Soc., 2008, 130, 11510-11517. 